\title{
Utility of a Multiparametric Quantitative MRI Model That Assesses Myelin and Edema for Evaluating Plaques, Periplaque White Matter, and Normal-Appearing White Matter in Patients with Multiple Sclerosis: A Feasibility Study
}

\author{
(D) A. Hagiwara, (D) M. Hori, (DK. Yokoyama, (D) M.Y. Takemura, (D). Andica, (DK.K. Kumamaru, (D) M. Nakazawa, (D) N. Takano,
} (D) H. Kawasaki, DS. Sato, (D) N. Hamasaki, (D) A. Kunimatsu, and (DS. Aoki

\begin{abstract}
BACKGROUND AND PURPOSE: T1 and T2 values and proton density can now be quantified on the basis of a single MR acquisition. The myelin and edema in a voxel can also be estimated from these values. The purpose of this study was to evaluate a multiparametric quantitative MR imaging model that assesses myelin and edema for characterizing plaques, periplaque white matter, and normal-appearing white matter in patients with MS.
\end{abstract}

MATERIALS AND METHODS: We examined 3T quantitative MR imaging data from 21 patients with MS. The myelin partial volume, excess parenchymal water partial volume, the inverse of $\mathrm{Tl}$ and transverse $\mathrm{T} 2$ relaxation times $(\mathrm{R} 1, \mathrm{R} 2)$, and proton density were compared among plaques, periplaque white matter, and normal-appearing white matter.

RESULTS: All metrics differed significantly across the 3 groups $(P<.001)$. Those in plaques differed most from those in normal-appearing white matter. The percentage changes of the metrics in plaques and periplaque white matter relative to normal-appearing white matter were significantly more different from zero for myelin partial volume (mean, $-61.59 \pm 20.28 \%$ [plaque relative to normal-appearing white matter], and mean, $-10.51 \pm 11.41 \%$ [periplaque white matter relative to normal-appearing white matter]), and excess parenchymal water partial volume $\left(13.82 \times 10^{3} \pm 49.47 \times 10^{3} \%\right.$ and $\left.51.33 \times 10^{2} \pm 155.31 \times 10^{2} \%\right)$ than for R1 $(-35.23 \pm 13.93 \%$ and $-6.08 \pm 8.66 \%)$, R2 (-21.06 \pm $11.39 \%$ and $-4.79 \pm 6.79 \%)$, and proton density $(23.37 \pm 10.30 \%$ and $3.37 \pm 4.24 \%)$.

CONCLUSIONS: Multiparametric quantitative MR imaging captures white matter damage in MS. Myelin partial volume and excess parenchymal water partial volume are more sensitive to the MS disease process than R1, R2, and proton density.

ABBREVIATIONS: EDSS = Expanded Disability Status Scale; NAWM = normal-appearing white matter; PD = proton density; PWM = periplaque white matter; $\mathrm{V}_{\mathrm{CL}}=$ cellular partial volume; $\mathrm{V}_{\mathrm{EPW}}=$ excess parenchymal water partial volume; $\mathrm{V}_{\mathrm{FW}}=$ free water partial volume; $\mathrm{V}_{\mathrm{MY}}=$ myelin partial volume; \%BPV $\mathrm{FPW}_{\mathrm{EP}}=$ the percentage of excess parenchymal water volume in brain parenchyma; \%BPV $\mathrm{MY}=$ the percentage of myelin volume in brain parenchyma; $\mathrm{QRAPMASTER}=$ quantification of relaxation times and proton density by multiecho acquisition of a saturation-recovery using turbo spin-echo readout; $\mathrm{R} 1$ and $\mathrm{R} 2=\mathrm{inverse}$ of $\mathrm{Tl}$ and transverse T2 relaxation times

Received June 13, 2016; accepted after revision August 29.

From the Department of Radiology (A.H., A.K.), Graduate School of Medicine, University of Tokyo, Tokyo, Japan; Departments of Radiology (A.H., M.H., M.Y.T., C.A., K.K.K., M.N., N.T., H.K., S.S., N.H., S.A.) and Neurology (K.Y.), Juntendo University School of Medicine, Tokyo, Japan; and Department of Radiological Sciences (M.N.), Graduate School of Human Health Sciences, Tokyo Metropolitan University, Tokyo, Japan. This work was supported by: the Japan Society for the Promotion of Science, KAKENHI grant No. 16K19852; the program for Brain Mapping by Integrated Neurotechnologies for Disease Studies (Brain/MINDS) from Japan Agency for Medical Research and Development (AMED); and a Grant-in-Aid for Scientific Research on Innovative Areas (ABiS) from the Ministry of Education, Culture, Sports, Science and Technology of Japan. This work was funded by the Impulsing Paradigm Change through disruptive Technologies (ImPACT) Program of Council for Science, Technology and Innovation (Cabinet Office, Government of Japan).

Please address correspondence to Akifumi Hagiwara, MD, Department of Radiology, Graduate School of Medicine, University of Tokyo, 7-3-1, Hongo, Bunkyo-ku, Tokyo, Japan, 113-8655; e-mail: ahagiwara-tky@umin.ac.jp

- Indicates open access to non-subscribers at www.ajnr.org aging plays a major role in the diagnosis and surveillance of patients with MS for initial and follow-up detection of focal cerebral lesions. ${ }^{1}$ In addition to conventional MR imaging techniques including T2-weighted imaging, quantitative MR imaging techniques enable characterization of MS lesions and detection of otherwise hidden abnormalities in normal-appearing white matter (NAWM) ${ }^{2,3}$ Moreover, diffusion tensor imaging and $q$-space imaging reveal abnormalities of white matter at the periphery of visible plaques on conventional MR images (periplaque white matter $[\mathrm{PWM}]$ ) and $\mathrm{NAWM}^{4,5}$ : The fractional anisotropy and apparent diffusion coefficient measured by diffusion tensor imaging and root mean square displacement measured by $q$-space imaging were worst in plaques, and in PWM, worse than in NAWM.

A recently developed MR imaging quantification pulse se-

http://dx.doi.org/10.3174/ajnr.A4977 
quence, QRAPMASTER (quantification of relaxation times and proton density by multiecho acquisition of a saturation-recovery using turbo spin-echo readout), has made it possible to quantify longitudinal $\mathrm{T} 1$ and transverse $\mathrm{T} 2$ relaxation times, their inverses $\mathrm{R} 1$ and $\mathrm{R} 2$, and proton density (PD) in a single acquisition in a clinically acceptable time. ${ }^{6}$ A phantom study has shown that these measurements are sufficiently accurate and reproducible for use in clinical practice. ${ }^{7}$ Several studies have also shown the validity of this sequence in evaluating diseases such as metastatic brain tumors ${ }^{8}$ and Sturge-Weber syndrome, ${ }^{9,10}$ in addition to MS. ${ }^{11}$ Even though the synthetic FLAIR image has lower image quality than the conventional FLAIR image, synthetic MR imaging overall has been shown to have comparable diagnostic power with conventional MR imaging for MS, while additionally offering fast and robust volumetry. ${ }^{11}$ By using the QRAPMASTER pulse sequence, the R1, R2, and PD values of plaques, NAWM, and diffusely abnormal white matter of patients with MS were shown to be different from those of white matter in healthy controls. ${ }^{12}$ Furthermore, the myelin partial volume $\left(\mathrm{V}_{\mathrm{MY}}\right)$ and excess parenchymal water partial volume $\left(\mathrm{V}_{\mathrm{EPW}}\right)$ can now be estimated from $\mathrm{R} 1, \mathrm{R} 2$, and $\mathrm{PD},{ }^{13}$ to indicate the quantities of myelin and edema, respectively, in the brain. In the pathologic brain, a decrease in $V_{M Y}$ indicating myelin loss or an increase in $\mathrm{V}_{\mathrm{EPW}}$ indicating edema will occur in this model. This model postulates 4 partial volume compartments: $V_{M Y}$, the cellular partial volume $\left(V_{C L}\right)$, the free water partial volume $\left(\mathrm{V}_{\mathrm{FW}}\right)$, and $\mathrm{V}_{\mathrm{EPW}}$. The model assumes that each compartment has its own R1, R2, and PD values and that the relaxation behaviors of all 4 compartments contribute to the effective R1, R2, and PD values of an acquisition voxel. $V_{M Y}$ contains myelin water and myelin macromolecules. $\mathrm{V}_{\mathrm{CL}}$ contains intracellular water, extracellular water, and nonmyelin macromolecules. Myelin water is trapped between myelin sheaths and has a much shorter T2 relaxation time than intracellular and extracellular water. The commonly calculated myelin water fraction corresponds to $\mathrm{PD}$ in the $\mathrm{V}_{\mathrm{MY}}$. The proportional relation between myelin water fraction and myelin content has been validated by histopathology. ${ }^{14,15} \mathrm{~V}_{\mathrm{EPW}}$, or edema, adds water to the $\mathrm{V}_{\mathrm{CL}}$. Because no distinction can be made between excess water and the already present water in the $\mathrm{V}_{\mathrm{CL}}$, the magnetization exchange rate between $V_{E P W}$ and $V_{C L}$ is assumed to be infinitely high. $V_{M Y}$ and $\mathrm{V}_{\mathrm{EPW}}$ may reflect the disease burden of patients with MS more specifically than R1, R2, and PD.

The aim of this study was to evaluate this multiparametric quantitative MR imaging model that assesses myelin and edema for characterizing plaques, PWM, and NAWM in patients with MS.

\section{MATERIALS AND METHODS Study Participants}

The present study was approved by the institutional review board of Juntendo University Hospital, Japan. Given its retrospective nature, written informed consent was waived. All patient information was anonymized and de-identified before analysis. Data from 36 consecutive patients with MS who underwent quantitative MR imaging from April 2015 through November 2015 were retrospectively reviewed. These patients were diagnosed according to standard criteria. ${ }^{1,16,17}$ Of the 36 patients, 15 had diffusely abnormal white matter ${ }^{18}$ and were excluded from the study be- cause it was difficult to adequately evaluate MS focal lesions in these patients. Therefore, 21 patients ( 4 males and 17 females; mean age, 38.3 years; age range, $16-61$ years) were included in the analysis. Of these, 18 had relapsing-remitting MS and 3 had clinically isolated syndrome. The mean score on the Expanded Disability Status Scale (EDSS) ${ }^{19}$ at image acquisition was 0.8 (range, $0-6.0$ ), and the mean disease duration was $7.1 \pm 4.8$ years. With the exception of 1 plaque in 1 patient, no new lesion was detected on conventional MR imaging since the last ones obtained at least 4 months earlier. All patients were clinically stable for at least 6 months, except 1 who presumably had optic neuritis 2 months before MR imaging but showed no new lesion on conventional MR imaging.

\section{MR Imaging}

All MR imaging sequences were performed on a 3T scanner (Discovery MR750w; GE Healthcare, Milwaukee, Wisconsin) with a 12-channel head coil. All patients underwent quantitative axial MR imaging and conventional axial T1-weighted inversion recovery, T2-weighted, and FLAIR imaging.

Quantitative MR imaging was performed by using the 2D axial QRAPMASTER pulse sequence. ${ }^{6}$ This pulse sequence is a multisection, multiecho, multisaturation delay method of saturationrecovery acquisition by a turbo spin-echo readout, with which images are obtained by different combinations of TE and saturation delay time. In this study, 2 sets of TE values and 4 sets of delay time values were used to generate 8 real images and 8 imaginary images in 1 section to quantify longitudinal T1 and transverse $\mathrm{T} 2$ relaxation times as well as the $\mathrm{PD}$. The TE values used were 16.9 and $84.5 \mathrm{~ms}$, and the delay time was set as defined by the manufacturer (SyntheticMR, Linköping, Sweden). The parameters used for quantitative MR imaging were as follows: FOV, $240 \times 240 \mathrm{~mm}$; matrix, $320 \times 320$; echo-train length, 10 ; bandwidth, $31.25 \mathrm{kHz}$; section thickness/gap, 4.0/1.0 mm; number of sections, 30 .

On the basis of the assumption that R1, R2, and PD values of $\mathrm{V}_{\mathrm{MY}}, \mathrm{V}_{\mathrm{EPW}}, \mathrm{V}_{\mathrm{CL}}$, and $\mathrm{V}_{\mathrm{FW}}$ all contribute to the effective R1, R2, and $\mathrm{PD}$ in a voxel, a model was created to estimate partial volumes of these 4 compartments by running Bloch equations and setting up a cost function in a spatially normalized and averaged brain from a group of 20 healthy controls described by Warntjes et al. ${ }^{13}$ Using this model, we created $\mathrm{V}_{\mathrm{MY}}$ and $\mathrm{V}_{\mathrm{EPW}}$ maps from R1, R2, and PD maps. This procedure was automatically performed by SyMRI software (Version 8.0; SyntheticMR). The R1, R2, and PD maps were then used to create synthetic MR images. Conventional T1-weighted inversion recovery images were obtained by using the following parameters: TR, $3294 \mathrm{~ms}$; TE, $18 \mathrm{~ms}$; TI, 908 $\mathrm{ms}$; FOV , $240 \times 216 \mathrm{~mm}$; matrix, $352 \times 256$; echo-train length, 8 ; section thickness/gap, 4.0/1.0 mm; number of sections, 30. T2weighted images were obtained by using TR, $4500 \mathrm{~ms}$; TE, $111 \mathrm{~ms}$; FOV, $240 \times 240 \mathrm{~mm}$; matrix, $512 \times 512$; echo-train length, 24 ; section thickness/gap, 4.0/1.0 mm; number of sections, 30. FLAIR images were obtained by using TR, 9000 ms; TE, 124 ms; TI, 2472 ms; FOV, $240 \times 240 \mathrm{~mm}$; matrix, $320 \times 224$; echo-train length, 16; section thickness/gap, 4.0/1.0 mm; number of sections, 30 . Conventional MR images were obtained at the same sections as the quantitative MR images. 


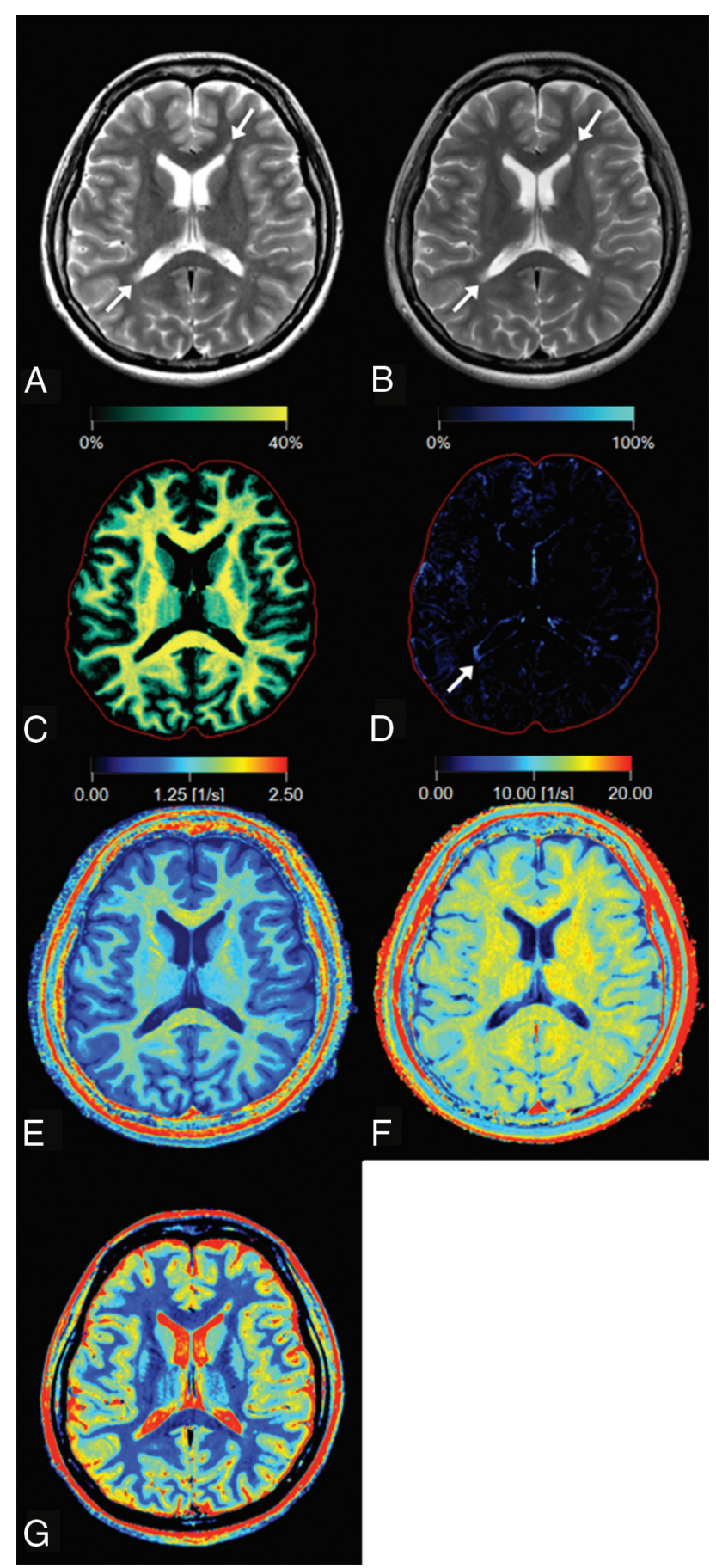

FIG 1. Representative images of a 27-year-old woman with multiple sclerosis. Panels show a synthetic T2-weighted image (A), a conventional T2-weighted image $(B)$, and maps of myelin partial volume $(C)$, excess parenchymal water partial volume $(D)$, Rl $(E)$, R2 $(F)$, and PD $(G)$. Two plaques are shown by arrows on the T2-weighted images ( $A$ and $B)$. On the $V_{E P W}$ map $(D)$, the periphery of the plaque adjacent to the trigone of the right ventricle (arrow) is visible but the one adjacent to the anterior horn of the left ventricle is not. The $\mathrm{V}_{\text {EPW }}$ of this invisible plaque was very low but still higher than that of NAWM. The red intracranial outline is displayed for visual guidance in tissue images $(C$ and $D)$.

\section{Image Analysis}

Synthetic T2-weighted images and maps of $\mathrm{V}_{\mathrm{MY}}, \mathrm{V}_{\mathrm{EPW}}, \mathrm{R} 1, \mathrm{R} 2$, and PD were created from raw quantification data by the SyMRI software on a commercial personal computer and converted to

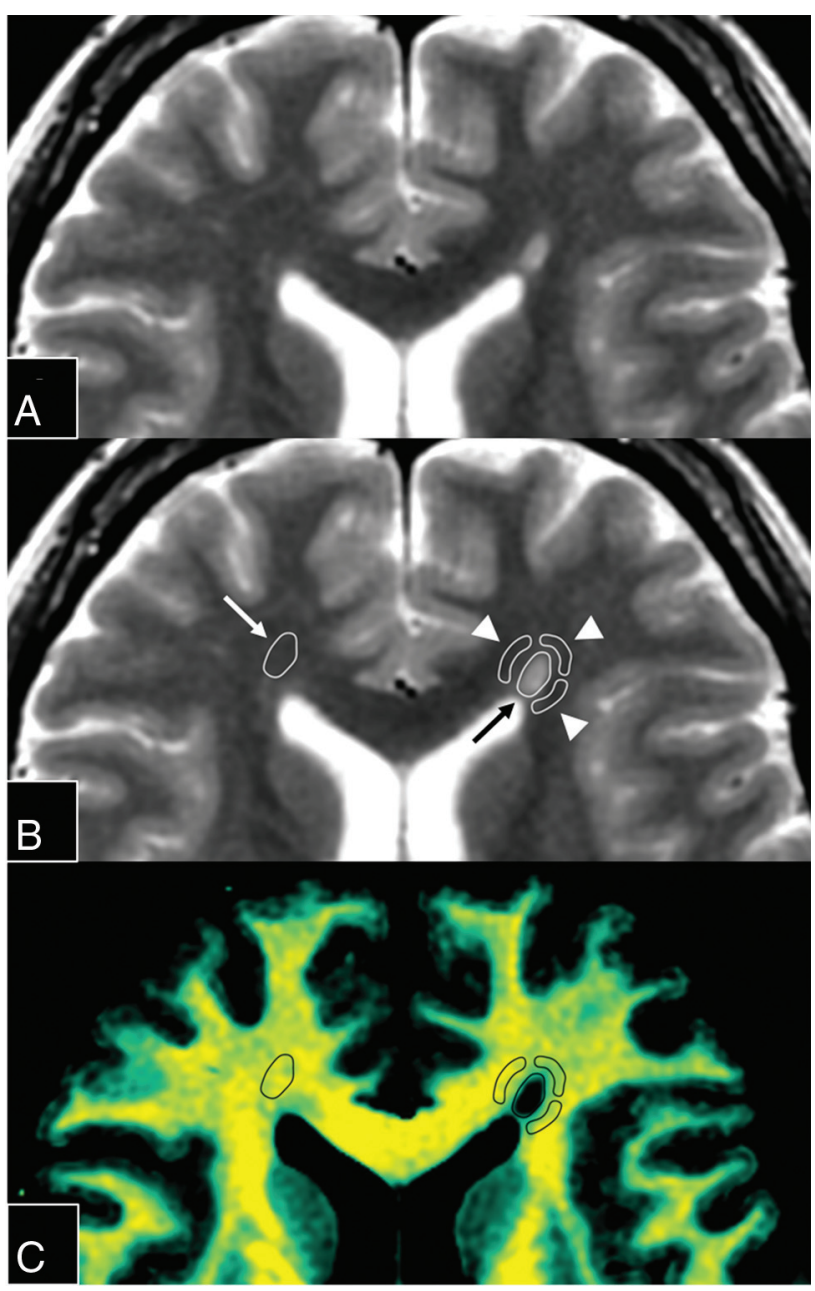

FIG 2. Magnified images of Fig $1 A$. The upper 2 panels show the same synthetic T2-weighted image without $(A)$ or with $(B)$ placement of ROIs. An ROI (black arrow) was drawn on a plaque adjacent to the left anterior horn, and 3 ROls (arrowheads) were placed on periplaque white matter to encircle the plaque. The fourth ROI on PWM was discarded because it overlapped the CSF. The ROI of the plaque was copied and pasted onto the contralateral normal-appearing white matter (white arrow). These ROls were then copied and pasted onto each quantification map. A map of the corresponding myelin partial volume $(C)$ is shown as an example.

DICOM files (Fig 1). Synthetic T2-weighted images were produced by using the following parameters: TR, $4500 \mathrm{~ms}$; TE, 100 $\mathrm{ms}$. These data were then analyzed by OsiriX Imaging Software, Version 7.0.3 (http:// www.osirix-viewer.com). ROIs were drawn on plaques, PWM, and NAWM on synthetic T2-weighted images. A plaque was defined as an area of abnormally high intensity, $>5$ $\mathrm{mm}$, on a T2-weighted image; PWM was defined as a normalintensity white-matter area closest to a plaque; and NAWM was defined as a normal-intensity area contralateral to a plaque. ${ }^{4,5} \mathrm{An}$ experienced neuroradiologist (A.H.) used conventional and synthetic images to confirm 135 plaques, which were then analyzed. A single investigator (M.N.), blinded to the clinical information, manually placed ROIs on T2-weighted images. A freehand ROI was drawn to encircle a plaque, after which up to 4 ROIs, approximately half the size of the initial ROI, were placed on the PWM of that plaque (Fig 2). The PWM ROIs were placed so that adjacent ROIs were approximately $90^{\circ}$ apart to form a circle that sur- 
rounded the plaque. A PWM ROI that overlapped CSF, gray matter, or other plaques was removed. Consequently, 128 PWM ROIs were discarded on this basis. The mean ROI size was $44.82 \pm$ $29.12 \mathrm{~mm}^{2}$ for a plaque and $21.19 \pm 12.59 \mathrm{~mm}^{2}$ for PWM. The ROI of a plaque was copied and pasted onto the contralateral NAWM. Finally, these ROIs were copied and pasted onto the maps of $\mathrm{V}_{\mathrm{MY}}, \mathrm{V}_{\mathrm{EPW}}, \mathrm{R} 1, \mathrm{R} 2$, and $\mathrm{PD}$ in the same patient, and the mean value of each ROI was recorded. The percentages of myelin and excess parenchymal water volume in brain parenchyma $\left(\% \mathrm{BPV}_{\mathrm{MY}}\right.$ and $\left.\% B P V_{\mathrm{EPW}}\right)$ were also calculated on SyMRI software and recorded.

\section{Statistical Analysis}

Statistical analysis was conducted with the software package R, Version 3.2.1 (http://www.R-project.org/). Not all data were normally distributed; therefore, we used the Steel-Dwass test, which is a nonparametric test for multiple comparisons, to compare the values of $\mathrm{V}_{\mathrm{MY}}, \mathrm{V}_{\mathrm{EPW}}, \mathrm{R} 1, \mathrm{R} 2$, and $\mathrm{PD}$ among plaques, $\mathrm{PWM}$, and NAWM. The percentage change of plaques or PWM relative to NAWM was also calculated and compared among different metrics (ie, $V_{M Y}, V_{E P W}, R 1, R 2$, and $P D$ ). The sign of this percentage change for $\mathrm{V}_{\mathrm{MY}}, \mathrm{R} 1$, and $\mathrm{R} 2$ was inverted for statistical analysis because overall, the values of these metrics were higher in NAWM than in plaques or PWM. EDSS and disease duration were correlated with $\% \mathrm{BPV}_{\mathrm{MY}}$ and $\% \mathrm{BPV}_{\mathrm{EPW}}$ by using the Spearman rank order correlation coefficient. A 2 -sided $P$ value $<.05$ was considered significant.

\section{RESULTS}

The results of ROI analysis and comparisons among plaques, PWM, and NAWM are shown in Table 1. All $V_{\text {MY }}, V_{\text {EPW }}, \mathrm{R} 1, \mathrm{R} 2$, and PD values differed significantly among plaques, PWM, and NAWM. $V_{M Y}$ was lower in plaques and PWM than in NAWM, with plaques showing the lowest value; $V_{E P W}$ was higher in plaques and PWM than in NAWM, with plaques showing the highest value; R1 was lower in plaques and PWM than in NAWM, with plaques showing the lowest value; R2 was lower in plaques and PWM than in NAWM, with plaques showing the lowest value; and PD was higher in plaques and PWM than in NAWM, with plaques showing the highest value.

\begin{tabular}{|c|c|c|c|c|c|}
\hline & $\mathrm{V}_{\mathrm{MY}}(\%)$ & $\mathrm{V}_{\text {EPW }}(\%)$ & $\mathrm{R} 1\left(\mathrm{~s}^{-1}\right)$ & $R 2\left(s^{-1}\right)$ & PD (\%) \\
\hline Plaques & $12.59 \pm 6.66$ & $5.82 \pm 4.75$ & $0.90 \pm 0.20$ & $10.88 \pm 1.41$ & $78.86 \pm 6.35$ \\
\hline PWM & $29.29 \pm 3.73$ & $2.31 \pm 2.38$ & $1.31 \pm 0.13$ & $13.14 \pm 0.77$ & $68.09 \pm 2.49$ \\
\hline NAWM & $32.88 \pm 3.12$ & $0.92 \pm 1.90$ & $1.40 \pm 0.08$ & $13.85 \pm 0.97$ & $63.97 \pm 2.07$ \\
\hline
\end{tabular}

${ }^{a}$ Values are mean \pm SD. $P<.001$ for all metrics among each tissue type.

Table 2: Percentage changes of $\mathrm{V}_{\mathrm{MY}}, \mathrm{V}_{\mathrm{EPW}}, \mathrm{R} 1, \mathrm{R2}$, and PD in plaques and periplaque white matter relative to normal-appearing white matter $^{\mathrm{a}}$

\begin{tabular}{lccccc}
\hline & $\mathbf{V}_{\text {MY }}(\%)$ & $\mathbf{V}_{\text {EPW }}(\%)$ & R1 (\%) & R2 (\%) & PD (\%) \\
\hline Plaques & $-61.59 \pm 20.28^{\mathrm{b}}$ & $13.82 \times 10^{3} \pm 49.47 \times 10^{3 \mathrm{~b}}$ & $-35.23 \pm 13.93^{\mathrm{b}}$ & $-21.06 \pm 11.39^{\mathrm{b}}$ & $23.37 \pm 10.30^{\mathrm{b}}$ \\
PWM & $-10.51 \pm 11.41^{\mathrm{c}}$ & $51.33 \times 10^{2} \pm 155.31 \times 10^{2 \mathrm{c}}$ & $-6.08 \pm 8.66^{\mathrm{c}}$ & $-4.79 \pm 6.79^{\mathrm{c}}$ & $3.37 \pm 4.24^{\mathrm{b}}$ \\
\hline
\end{tabular}

${ }^{a}$ Values are mean \pm SD. Of the 135 ROIs, 39 were discarded after calculating the percentage change of $V_{E P W}$ relative to NAWM because the $V_{E P W}$ of these ROIs was equivalent to zero in NAWM.

${ }^{b} P<.001$ in the percentage change for plaques relative to NAWM for comparison between each pair of metrics, except between R2 and PD ( $\left.P=.31\right)$. Statistical analysis was performed for absolute values.

${ }^{c} P<.001$ in the percentage change for PWM relative to NAWM between $\mathrm{V}_{\mathrm{EPW}}$ and other metrics, and between $\mathrm{V}_{\mathrm{MY}}$ and $\mathrm{R} 2$ or $\mathrm{PD}, P<.05$ between $\mathrm{V}_{\mathrm{MY}}$ and R1, and $P>.05$ between R2 and R1 (.31) or PD (.30). Statistical analysis was performed for absolute values. 
more in plaques. ${ }^{21}$ It is suggested that this upregulation is for protecting damaged tissue from disturbed water balance. Our result of elevated $\mathrm{V}_{\mathrm{EPW}}$ in these regions supports this speculation. Because MR spectroscopy results suggest that acute lesions are more edematous than chronic lesions, ${ }^{27} \mathrm{~V}_{\mathrm{EPW}}$ in a plaque may predict the acute status of a lesion (ie, its enhancement). Visual inspection of Fig $1 D$ (arrow) reminds us of a ring-pattern enhancement with the $\mathrm{V}_{\mathrm{EPW}}$ for a plaque higher in its periphery than in its center. Given that blood-brain barrier disruption and edema formation are correlated phenomena, ${ }^{28}$ a higher $\mathrm{V}_{\mathrm{EPW}}$ may suggest the existence of blood-brain barrier disruption. Although R1, $\mathrm{R} 2$, and PD are good predictors of lesion enhancement, ${ }^{29}$ the combination of $\mathrm{V}_{\mathrm{MY}}$ and $\mathrm{V}_{\mathrm{EPW}}$ may be a better predictor because it provides a more specific description of a lesion. This conjecture was not validated here because almost all lesions investigated were chronic and no contrast medium was used. In this study, $\mathrm{V}_{\mathrm{MY}}$ and $\mathrm{V}_{\mathrm{EPW}}$ were more sensitive in showing abnormalities in plaques and PWM than R1, R2, and PD. Therefore, $\mathrm{V}_{\mathrm{MY}}$ and $\mathrm{V}_{\mathrm{EPW}}$ are potentially more sensitive biomarkers of the disease process than R1, R2, and PD, especially in patients with MS.

Radiologic-pathologic correlations of plaques, diffusely abnormal white matter, and NAWM have been well-established, with axonal loss and decreased myelin density most severe in plaques and more severe in diffusely abnormal white matter than in NAWM. ${ }^{18}$ Even though PWM has been investigated radiologically ${ }^{4,5}$ and pathologically ${ }^{21}$ so far, currently no study has correlated the normal-appearing PWM on T2-weighted images with histology. Alterations of astrocyte functions in PWM have been demonstrated, which are accompanied by low-grade inflammation and a progressive loss of myelin without sufficient remyelination. $^{21}$ Future study should investigate normal-appearing PWM on T2-weighted images histologically, which we investigated by multiparametric MR imaging in this study.

No significant correlations with EDSS and disease duration were found with $\% \mathrm{BPV}_{\mathrm{MY}}$ and $\% \mathrm{BPV}_{\mathrm{EPW}}$ in this study. The correlation between the myelin water fraction, which is the $\mathrm{PD}$ of $\mathrm{V}_{\mathrm{MY}}$ investigated in this study, of NAWM and EDSS has been shown in primary-progressive $\mathrm{MS}^{30}$ but not in relapsing-remitting MS so far. ${ }^{31}$ These investigations suggest that the myelin content in the NAWM of the severe progressive form of MS (ie, primary-progressive MS) correlates more with EDSS than that of the less progressive form (ie, relapsing-remitting MS), which was investigated in this study. This conjecture should be validated in a larger study that includes both subtypes of patients with MS and uses a single method of measuring myelin water fraction or $\mathrm{V}_{\mathrm{MY}}$.

There are a number of potential limitations to our study. First, our study included a small number of patients and did not include healthy controls. Second, the age and disease burdens of the patients varied widely; consequently, the specific pathology of plaques, PWM, and NAWM may have been diverse. This problem could be resolved in the future by studying a large population stratified by age and disease burden. Third, the multiparametric model used in this study was trained only for healthy brains. It needs validation in several studies, including our current one, and further refinement for patients with brain diseases. Last, although the multiparametric quantitative MR imaging model used in this study represented the amount of myelin by $\mathrm{V}_{\mathrm{MY}}$, axonal status was not specifically investigated. The axonal volume fraction can now be estimated from neurite orientation dispersion and density imaging and the myelin volume fraction. ${ }^{32,33}$ Therefore, the axonal volume fraction will be combined with the myelin partial volume to further clarify the MS disease process in a future study.

\section{CONCLUSIONS}

$\mathrm{V}_{\mathrm{MY}}, \mathrm{V}_{\mathrm{EPW}}, \mathrm{R} 1, \mathrm{R} 2$, and $\mathrm{PD}$ were more abnormal in plaques and PWM than in NAWM, with plaques showing the most abnormal values. $\mathrm{V}_{\mathrm{MY}}$ and $\mathrm{V}_{\mathrm{EPW}}$ were more sensitive to the MS disease process than $\mathrm{R} 1, \mathrm{R} 2$, and $\mathrm{PD} . \mathrm{V}_{\mathrm{MY}}$ and $\mathrm{V}_{\mathrm{EPW}}$ are useful estimators of disease burden in patients with MS.

Disclosures: Akifumi Hagiwara—RELATED: Grant:Japan Society for the Promotion of Science; Nao Takano-UNRELATED: Payment for Lectures including Service on Speakers Bureaus: GE Healthcare Japan, Comments: The 42nd Japanese Society of Radiological Technology Autumn Scientific Congress, October 9-11, 2014, Sapporo, Hokkaido, Japan: luncheon seminar "Experience of MR Angiography (Silent MRA) using the SILENZ Sequence." Akira Kunimatsu-UNRELATED: Payment for Lectures including Service on Speakers Bureaus: Siemens Japan, Toshiba, Philips Electronics Japan, Terumo; Payment for Manuscript Preparation: Bayer Yakuhin, GE Healthcare Japan. Shigeki Aoki—RELATED: Grant: Impulsing Paradigm Change through Disruptive Technologies (ImPACT) program of Council for Science, Technology and Innovation (Cabinet Office, Government of Japan), program for Brain Mapping by Integrated Neurotechnologies for Disease Studies (Brain/MINDS) from Japan Agency for Medical Research and development (AMED), Grant-in-Aid for Scientific Research on Innovative Areas (ABiS) from the Ministry of Education, Culture, Sports, Science and Technology of Japan. * Money paid to the institution.

\section{REFERENCES}

1. Polman CH, Reingold SC, Banwell B, et al. Diagnostic criteria for multiple sclerosis: 2010 revisions to the McDonald criteria. Ann Neurol 2011;69:292-302 CrossRef Medline

2. Filippi M, Rocca MA, De Stefano N, et al. Magnetic resonance techniques in multiple sclerosis: the present and the future. Arch Neurol 2011;68:1514-20 CrossRef Medline

3. Giorgio A, De Stefano N. Advanced structural and functional brain MRI in multiple sclerosis. Semin Neurol 2016;36:163-76 CrossRef Medline

4. Guo AC, MacFall JR, Provenzale JM. Multiple sclerosis: diffusion tensor MR imaging for evaluation of normal-appearing white matter. Radiology 2002;222:729-36 CrossRef Medline

5. Hori M, Yoshida M, Yokoyama K, et al. Multiple sclerosis: benefits of q-space imaging in evaluation of normal-appearing and periplaque white matter. Magn Reson Imaging 2014;32:625-29 CrossRef Medline

6. Warntjes JB, Leinhard OD, West J, et al. Rapid magnetic resonance quantification on the brain: optimization for clinical usage. Magn Reson Med 2008;60:320-29 CrossRef Medline

7. Krauss W, Gunnarsson M, Andersson T, et al. Accuracy and reproducibility of a quantitative magnetic resonance imaging method for concurrent measurements of tissue relaxation times and proton density. Magn Reson Imaging 2015;33:584-91 CrossRef Medline

8. Hagiwara A, Hori M, Suzuki M, et al. Contrast-enhanced synthetic MRI for the detection of brain metastases. Acta Radiol Open 2016;5: 2058460115626757 CrossRef Medline

9. Hagiwara A, Nakazawa M, Andica C, et al. Dural enhancement in a patient with Sturge-Weber syndrome revealed by double inversion recovery contrast using synthetic MRI. Magn Reson Med Sci 2016; 15:151-52 CrossRef Medline

10. Andica C, Hagiwara A, Nakazawa M, et al. The advantage of synthetic MRI for the visualization of early white matter change in an infant with Sturge-Weber syndrome. Magn Reson Med Sci 2016 Mar 21. [Epub ahead of print] Medline

AJNR Am J Neuroradiol 38:237-42 Feb 2017 www.ajnr.org 
11. Granberg T, Uppman M, Hashim F, et al. Clinical feasibility of synthetic MRI in multiple sclerosis: a diagnostic and volumetric validation study. AJNR Am J Neuroradiol 2016;37:1023-29 CrossRef Medline

12. West J, Aalto A, Tisell A, et al. Normal appearing and diffusely abnormal white matter in patients with multiple sclerosis assessed with quantitative MR. PLoS One 2014;9:e95161 CrossRef Medline

13. Warntjes M, Engström M, Tisell A, et al. Modeling the presence of myelin and edema in the brain based on multi-parametric quantitative MRI. Front Neurol 2016;7:16 CrossRef Medline

14. Bjarnason TA, Vavasour IM, Chia CL, et al. Characterization of the NMR behavior of white matter in bovine brain. Magn Reson Med 2005;54:1072-81 CrossRef Medline

15. Laule C, Leung E, Lis DK, et al. Myelin water imaging in multiple sclerosis: quantitative correlations with histopathology. Mult Scler 2006;12:747-53 CrossRef Medline

16. McDonald WI, Compston A, Edan G, et al. Recommended diagnostic criteria for multiple sclerosis: guidelines from the International Panel on the Diagnosis of Multiple Sclerosis. Ann Neurol 2001;50: 121-27 CrossRef Medline

17. Polman CH, Reingold SC, Edan G, et al. Diagnostic criteria for multiple sclerosis: 2005 revisions to the "McDonald Criteria." Ann Neurol 2005;58:840-46 CrossRef Medline

18. Seewann A, Vrenken H, van der Valk P, et al. Diffusely abnormal white matter in chronic multiple sclerosis: imaging and histopathologic analysis. Arch Neurol 2009;66:601-09 Medline

19. Kurtzke JF. A new scale for evaluating disability in multiple sclerosis. Neurology 1955;5:580 - 83 CrossRef Medline

20. Nijeholt GJ, Bergers E, Kamphorst W, et al. Post-mortem high-resolution MRI of the spinal cord in multiple sclerosis: a correlative study with conventional MRI, histopathology and clinical phenotype. Brain 2001;124:154-66 CrossRef Medline

21. Lieury A, Chanal M, Androdias G, et al. Tissue remodeling in periplaque regions of multiple sclerosis spinal cord lesions. Glia 2014;62:1645-58 CrossRef Medline

22. Narayanan S, Fu L, Pioro E, et al. Imaging of axonal damage in mul- tiple sclerosis: spatial distribution of magnetic resonance imaging lesions. Ann Neurol 1997;41:385-91 CrossRef Medline

23. Perry VH, Anthony DC. Axon damage and repair in multiple sclerosis. Philos Trans R Soc Lond B Biol Sci 1999;354:1641-47 CrossRef Medline

24. Dziedzic T, Metz I, Dallenga T, et al. Wallerian degeneration: a major component of early axonal pathology in multiple sclerosis. Brain Pathol 2010;20:976-85 CrossRef Medline

25. Simons M, Misgeld T, Kerschensteiner M. A unified cell biological perspective on axon-myelin injury. J Cell Biol 2014;206:335-45 CrossRef Medline

26. McDonald WI, Miller DH, Barnes D. The pathological evolution of multiple sclerosis. Neuropathol Appl Neurobiol 1992;18:319-34 CrossRef Medline

27. Helms G, Stawiarz L, Kivisakk P, et al. Regression analysis of metabolite concentrations estimated from localized proton MR spectra of active and chronic multiple sclerosis lesions. Magn Reson Med 2000; 43:102-10 Medline

28. Brosnan CF, Raine CS. The astrocyte in multiple sclerosis revisited. Glia 2013;61:453-65 CrossRef Medline

29. Blystad I, Hakansson I, Tisell A, et al. Quantitative MRI for analysis of active multiple sclerosis lesions without gadolinium-based contrast agent. AJNR Am J Neuroradiol 2016;37:94-100 CrossRef Medline

30. Kolind S, Matthews L, Johansen-Berg $\mathrm{H}$, et al. Myelin water imaging reflects clinical variability in multiple sclerosis. Neuroimage 2012; 60:263-70 CrossRef Medline

31. Faizy TD, Thaler C, Kumar D, et al. Heterogeneity of multiple sclerosis lesions in multislice myelin water imaging. PLoS One 2016;11: e0151496 CrossRef Medline

32. Zhang H, Schneider T, Wheeler-Kingshott CA, et al. NODDI: practical in vivo neurite orientation dispersion and density imaging of the human brain. Neuroimage 2012;61:1000-16 CrossRef Medline

33. Stikov N, Campbell JS, Stroh T, et al. In vivo histology of the myelin g-ratio with magnetic resonance imaging. Neuroimage 2015;118: 397-405 CrossRef Medline 